\title{
Combining Ability Analysis for Yield and its Components in Tomato (Solanum lycopersicum L.)
}

\author{
TA Vekariya ${ }^{1 *}$, GU Kulkarni ${ }^{1}$, DM Vekaria ${ }^{2}$, AP Dedaniya ${ }^{1}$ and JT Memon ${ }^{3}$ \\ ${ }^{1}$ Department of Genetics and Plant Breeding, College of Agriculture, Junagadh Agricultural University, Junagadh, Gujarat, India \\ ${ }^{2}$ Wheat Research Station, Junagadh Agricultural University, Junagadh, Gujarat, India \\ ${ }^{3}$ Department of Genetics and Plant Breeding, B. A. College of Agriculture, AAU, Anand, Gujarat, India
}

*Corresponding Author: TA Vekariya, Department of Genetics and Plant Breeding, College of Agriculture, Junagadh Agricultural University, Junagadh, Gujarat, India.

Received: April 23, 2019; Published: June 24, 2019

DOI: 10.31080 /ASAG.2019.03.0541

\begin{abstract}
The present investigation was undertaken to study the combining ability of parents and crosses for fruit yield and quality components in tomato using 40 hybrids involving 10 lines and 4 testers in line $\mathrm{x}$ tester fashion. Thus, the forty crosses along with their fourteen parents and one check variety (JT-3) consisted the experimental material for the present study. The genotypes were evaluated in randomized block design with 3 replications, at Vegetable Research Station, Junagadh Agricultural University, Junagadh, during Rabi 2017-18. The present study revealed that, analysis of variance for combing ability and the estimates of variance components indicated that the mean squares due to lines were significant for all characters which revealed significant contribution of lines towards general combining ability variance components for most of traits. The mean squares due to testers were also found significant for all the characters except number of fruits per cluster suggesting the larger contribution of testers towards component of gca variance. The mean sum of squares due to lines $\times$ testers interaction observed significant for all yield attributing traits which revealed the significant contribution of hybrids for specific combining ability variance components. This indicated the involvement of additive as well as non-additive type of gene actions in the inheritance of these characters. The best general combiners for various characters were GP-5 for plant height; GP-18 for number of branches per plant; GP-17 for number of fruits per cluster; GP-32 for days to $50 \%$ fruit set, days to $50 \%$ flowering, total number of fruits per plant, days to marketable maturity and number of clusters per plant; GP-39 for average fruit weight and number of locules per fruit; GP-12 for marketable yield per plant and pericarp thickness; GP-3 for total soluble solid. Best cross combinations viz., GP-5 × JT-3, GP-29 × AT-3 and GP-18 × AT-3 were found to be best specific combiners for marketable yield per plant.
\end{abstract}

Keywords: Solanum lycopersicum L; General Combining Ability; Specific Combining Ability

\section{Introduction}

Vegetables play an important role in the balanced diet by providing not only energy but also vital protective nutrients like vitamins, minerals and antioxidants "Eating of plateful vegetables keeps doctor away" is universal truth due to presence of phytochemicals like carotenoids, inosital phosphate, phytosterols etc. Tomato (Solanum lycopersicum L.) is one of the most important fruit vegetable, which has achieved tremendous popularity during the last century. Tomato is a member of the Solanaceae family. Its consumption has increased tremendously due to its multifarious uses like raw for salad, cooked as vegetable and processed in many forms as soup, sauces, ketchups, preserves, paste and puree [1].
The combining ability study is a powerful tool to discriminate good as well as poor combiners for choosing appropriate parental material in plant breeding programme. Moreover, information regarding general and specific combing ability enables the plant breeders to evaluate parental material and to decide a suitable breeding procedure for maximum character improvement. The knowledge of nature and magnitude of fixable and non-fixable types of gene effects governing the yield and its components is essential in order to formulate an efficient and a sound breeding programme to achieve the maximum genetic improvement in tomato. 


\section{Material and Methods}

The experimental material for the present study consists of 40 crosses (F1s) developed through line $\mathrm{x}$ tester mating design involving 10 lines and 4 testers. Ten lines (females) namely, GP-3, GP-5, GP-12, GP-14, GP-17, GP-18, GP-29, GP-32, GP-37, GP-39 and four testers (males) i.e. GT-1, JT-3, DVRT-2, AT-3 of tomato were selected on the basis of their phenotypic variability. Forty crosses were prepared during Rabi 2016-17 using ten lines as female and four testers as male. Thus, the forty crosses along with their fourteen parents and one check variety (JT-3) consisted the experimental material for the present study. The genotypes were evaluated in Randomized Block Design with 3 replications, at Vegetable Research Station, Junagadh Agricultural University, Junagadh, during Rabi $2017-18$. Geographically, Junagadh is situated at $21.5^{\circ} \mathrm{N}$ latitude and $70.5^{\circ} \mathrm{E}$ longitude with an altitude of 60 meters above the mean sea level. The soil of the experimental site is medium black, alluvial in origin and medium in organic matter.

\section{Results and Discussion}

With the advancement in biometrical genetics, several techniques are now available which permit analysis and selection of promising parents and crosses for further exploitation. Among them line $\times$ tester cross analysis is one of the principal techniques available for estimating combining ability when the genetic worth of relatively small number of parents are to be tested with great precision. Combining ability analysis is a powerful technique to discriminate good as well as poor combiners and for choosing appropriate parental material in the breeding programme of crop plants. At the same time, it also elucidates the nature of gene action involved in the inheritance of characters. The relative amount of gca and sca effects play a vital role in planning the appropriate and sound breeding programme. Therefore, the present investigation has been carried out to estimate general and specific combining ability of parents and hybrids, respectively and to know the type of gene action governing marketable yield and its component traits.

In autogamous crop species, the breeder is interested primarily in identifying the parental combinations that are likely to produce superior homologous lines. The possibility of such superior true breeding genotypes is supported by the preponderance of additive genetic variance. For improving yield potential of varieties and hybrids, decision should be made about the choice of right type of parents for hybridization. This is the importance of testing the parents for their combining ability because many times, high yielding parents may not combine well to give good segregants. The information on the type of gene action and combining ability of parents is helpful in planning future breeding programme.

General combining ability is attributed to additive genetic effect and additive $\times$ additive interaction effect and is, theoretically, fix- able. On the other hand, specific combining ability attributable to non-additive gene action may be due to either dominance or epistasis or both and is unfixable. The presence of non-additive genetic variance is the primary justification to initiate the hybridization programmed.

\section{Analysis of variance for combining ability}

The analysis of variance for combining ability and the estimates of variance components (Table 1 ) indicated that the mean squares due to lines were significant for all characters studied. This indicated significant contribution of lines towards general combining ability variance components for all the traits. The mean sums of squares due to testers were also significant for all the characters except number of fruits per cluster, suggesting larger contribution of testers towards component of general combining ability variance. The mean sum of squares due to line $\times$ testers interaction were significant for all the yield attributing traits which revealed the significant contribution of hybrids for specific combining ability variance components.

The magnitude of gca variance were higher than the sca variance for the characters, viz., days to $50 \%$ flowering, days to $50 \%$ fruit set, which indicated preponderance of additive gene action in the inheritance of these traits. Therefore, selection for these traits in early generations would be effective for developing the varieties in tomato breeding programme. This was further supported by high magnitude of $\sigma 2 \mathrm{gca} / \sigma 2 \mathrm{sca}$ ratios. Preponderance of additive variance in expression of these traits in tomato has also been reported by Sharma., et al. [2], Ahmed., et al. [3] and Singh., et al. [4] for days to $50 \%$ flowering.

The magnitude of sca variance were higher than gca variance for the characters, viz., plant height, number of branches per plant, number of clusters per plant, number of fruit per cluster, total number of fruits per plant, average fruit weight, number of locules per fruit, marketable yield per plant, pericarp thickness, fruit shape index, days to marketable maturity, number of cluster per plant, total soluble solid, acidity, which indicated preponderance of non-additive gene action in the inheritance of these traits. Therefore, heterosis breeding is used if commercially feasible. Similar findings were also reported by Gaikwad., et al. [5], Sharma., et al. [2], Ahmed., et al. [3] and Mali and Patel [6] for plant height (cm), Gaikwad., et al. [5] and Angadi., et al. [7] for number of branches per plant, Angadi., et al. [7] for number of clusters per plant, Gaikwad., et al. [5], Angadi., et al. [7] and Yadav., et al. [8] for number of fruits per plant, Gaikwad., et al. [5], Angadi., et al. [7], Agarwal., et al. [9] and El- Gabry., et al. [10] for average fruit weight (g), Gaikwad., et al. [5], Singh and Asati [11] and Kumari and Sharma [12] for marketable yield per plant, Gaikwad., et al. [5], Kumari and Sharma [12], for pericarp thickness, Ahmad., et al. [3] and Souza., et al. [13] for total soluble solids, Souza., et al. [13] and Shankar., et 


\begin{tabular}{|c|c|c|c|c|c|c|c|c|c|}
\hline Source & d.f. & $\begin{array}{c}\text { Days to } \\
\mathbf{5 0 \%} \\
\text { flowering }\end{array}$ & $\begin{array}{c}\text { Days to } \\
50 \% \\
\text { fruit set }\end{array}$ & $\begin{array}{l}\text { Plant } \\
\text { height }\end{array}$ & $\begin{array}{c}\text { No. of } \\
\text { Branches } \\
\text { per plant }\end{array}$ & $\begin{array}{l}\text { No. of } \\
\text { fruits per } \\
\text { cluster }\end{array}$ & $\begin{array}{l}\text { Total num- } \\
\text { ber of fruits } \\
\text { per plant }\end{array}$ & $\begin{array}{l}\text { Average fruit } \\
\text { weight }\end{array}$ & $\begin{array}{c}\text { No. of } \\
\text { locules per } \\
\text { fruit }\end{array}$ \\
\hline Replications & 2 & 5.108 & 4.633 & 17.034 & $1.304^{* *}$ & 0.506 & 2129.740 & 0.039 & 0.038 \\
\hline Lines & 9 & $35.670^{* *++}$ & $37.337^{* *++}$ & $4172.241^{* *}$ & $9.514^{* *}$ & $4.979 * *$ & $33063.030^{* *}$ & $1615.340^{* *++}$ & $1.391^{* *}$ \\
\hline Testers & 3 & $55.967^{* *}++$ & $70.030^{* *}++$ & $3342.910^{* *}$ & $18.302^{* *}$ & 0.271 & $39514.080 * *$ & $2033.100^{* *}+$ & $1.096^{* *}$ \\
\hline Lines $\times$ Testers & 27 & 7.435 & $8.826^{*}$ & $2287.760^{* *}$ & $9.726^{* *}$ & $4.107^{* *}$ & $14856.890^{* *}$ & $460.710^{* *}$ & $1.209 * *$ \\
\hline Error & 78 & 5.005 & 5.231 & 5.838 & 0.238 & 0.205 & 730.540 & 0.113 & 0.082 \\
\hline \multicolumn{10}{|c|}{$\begin{array}{c}\text { Variance } \\
\text { Components } \\
\end{array}$} \\
\hline \multicolumn{2}{|l|}{$\sigma^{2} l$} & $2.555^{* *}$ & $2.675^{* *}$ & 347.200 & 0.773 & 0.397 & 2694.370 & $134.602^{* *}$ & 0.109 \\
\hline \multicolumn{2}{|l|}{$\sigma^{2} t$} & $1.698^{* *}$ & $2.160^{* *}$ & 111.230 & 0.602 & 0.002 & 1292.780 & $67.766^{*}$ & 0.033 \\
\hline \multicolumn{2}{|l|}{$\sigma^{2}$ lt } & 0.810 & $1.198^{*}$ & $760.640^{* *}$ & $3.162^{* *}$ & $1.300^{* *}$ & $4708.780^{* *}$ & $153.533^{* *}$ & $0.375^{* *}$ \\
\hline \multicolumn{2}{|l|}{$\sigma^{2}$ gca } & $1.943^{* *}$ & $2.307^{* *}$ & $178.650^{* *}$ & $0.650^{*}$ & 0.115 & $1693.230^{* *}$ & $86.862^{* *}$ & 0.055 \\
\hline \multicolumn{2}{|l|}{$\sigma^{2}$ sca } & 0.810 & 1.198 & $760.640^{* *}$ & $3.162^{* *}$ & $1.300^{* *}$ & $4708.780^{* *}$ & $153.533^{* *}$ & $0.375^{* *}$ \\
\hline \multicolumn{2}{|c|}{$\sigma^{2}$ gca/ $\sigma^{2} \mathbf{s c a}$} & 2.399 & 1.925 & 0.234 & 0.205 & 0.088 & 0.360 & 0.565 & 0.147 \\
\hline
\end{tabular}

\begin{tabular}{|c|c|c|c|c|c|c|c|c|}
\hline Source & d.f. & $\begin{array}{c}\text { Marketable } \\
\text { yield per plant }\end{array}$ & $\begin{array}{c}\text { Pericarp } \\
\text { thickness }(\mathbf{m m})\end{array}$ & $\begin{array}{c}\text { Fruit shape } \\
\text { index }\end{array}$ & $\begin{array}{l}\text { Days to market- } \\
\text { able maturity }\end{array}$ & $\begin{array}{c}\text { No. of clusters } \\
\text { per plant }\end{array}$ & $\begin{array}{c}\text { Total Soluble } \\
\text { solids (\%) }\end{array}$ & $\begin{array}{c}\text { Acidity } \\
\text { (\%) }\end{array}$ \\
\hline Replications & 2 & $1.407^{*}$ & 0.007 & 0.001 & 0.325 & 19.989 & 0.114 & $0.013^{* *}$ \\
\hline Lines & 9 & $9.580^{* *}$ & $2.180^{* *}$ & $0.236^{* *}$ & $24.149^{* *}$ & $1261.588^{* *}+$ & $4.304^{* *}$ & $0.008^{* *}$ \\
\hline Testers & 3 & $22.087^{* *}$ & $1.317^{* *}$ & $0.511^{* *}$ & $46.808^{* *}+$ & $1850.112^{* *+}$ & $4.542^{* *}$ & $0.018^{* *}$ \\
\hline $\begin{array}{l}\text { Lines } x \\
\text { Testers }\end{array}$ & 27 & $13.672^{* *}$ & $1.278^{* *}$ & $0.206^{* *}$ & $12.833^{* *}$ & $533.135^{* *}$ & $2.858^{* *}$ & $0.010^{* *}$ \\
\hline Error & 78 & 0.315 & 0.009 & 0.004 & 0.897 & 12.280 & 0.0926 & 0.001 \\
\hline \multicolumn{9}{|c|}{$\begin{array}{c}\text { Variance } \\
\text { Components }\end{array}$} \\
\hline \multicolumn{2}{|l|}{$\sigma^{2} \mathrm{l}$} & 0.772 & 0.181 & 0.002 & 1.937 & $104.108^{*}$ & 0.351 & 0.001 \\
\hline \multicolumn{2}{|l|}{$\sigma^{2} \mathrm{t}$} & 0.725 & 0.043 & 0.002 & $1.530^{*}$ & $61.261^{*}$ & 0.148 & 0.001 \\
\hline \multicolumn{2}{|l|}{$\sigma^{2}$ lt } & $4.452^{* *}$ & $0.423^{* *}$ & $0.006^{* *}$ & $3.978^{* *}$ & $173.617^{* *}$ & $0.921^{* *}$ & $0.003^{* *}$ \\
\hline \multicolumn{2}{|l|}{$\boldsymbol{\sigma}^{2} \mathrm{gca}$} & 0.738 & $0.082^{*}$ & $0.002^{* *}$ & $1.646^{* *}$ & $73.503^{* *}$ & $0.206^{*}$ & 0.001 \\
\hline \multicolumn{2}{|l|}{$\boldsymbol{\sigma}^{2}$ sca } & $4.452^{* *}$ & $0.423^{* *}$ & $0.006^{* *}$ & $3.978^{* *}$ & $173.617^{* *}$ & $0.921^{* *}$ & $0.003^{* *}$ \\
\hline \multicolumn{2}{|c|}{$\sigma^{2}$ gca $/ \sigma^{2}$ sca } & 0.166 & 0.195 & 0.284 & 0.827 & 0.423 & 0.223 & 0.186 \\
\hline
\end{tabular}

Table 1: Analysis of variance for combining ability and variance components for different characters in tomato $*, * *$ Significant at $5 \%$ and $1 \%$ against error, respectively

,+++ Significant at $5 \%$ and $1 \%$ levels, respectively against line $\times$ tester interaction.

The estimation of genetic variance contributed by lines $(\sigma 2 \mathrm{l})$ and testers $(\sigma 2 \mathrm{t})$.

al. [14] for acidity. Gaikwad., et al. [5] and Tanvi., et al. [15] for average fruit weight (g). Angadi and Dharmati [7] and Souza., et al. [13] for number of locules per fruit.

In a view of these studies, it could be concluded that marketable yield is a complex character as compared to its components. Thus, as the quantitative character becomes complex, the contribution of non-additive gene action would be more. Under such situation, it would be worthwhile to resort to breeding methodologies, such as biparental mating and diallel selective mating than to conventional pedigree or backcross techniques which would leave the unfixable components of genetic variances which are usually exploited for yield and its components. 


\section{General and specific combining ability effects}

Selection of parents for hybridization programme is an important aspect in the crop improvement. In any sound breeding programme, the proper choice of parents based on their combining ability is a pre-requisite. Such studies not only provide necessary information regarding the choice of parent but, also illustrate the nature and magnitude of gene action involved in the inheritance of the characters of economics interest.

The combining ability analysis carried out to obtain useful information for selection of better parents and crosses for their future use in breeding programme. The information regarding nature and magnitude of gene action could also be obtained, which is useful in deciding breeding methodology aiming at exploitation of fixable (additive) and non-fixable (non-additive) genetic variances.

The summary of general combining ability effects (Table 2) of the parents revealed that none of the parents was found to be good general combiner for all the characters. General combining ability effects of the parents revealed that GP-12, GP-32 of the line and DVRT-2 of tester was found to be good general combiner for days to 50\% flowering and GP-12, GP-32 of lines and DVRT- 2 and JT-3 of testers was found to be good general combiner for days $50 \%$ fruit set. The good general combining ability effect was expressed by the female parent GP-3, GP-5, GP-14, GP-17 and GP-18 and male parent AT-3 for plant height. The estimate of general combining ability effect revealed that female parents GP-3, GP-18 and GP-29 and male parents JT-3 and AT-3 have good general combining ability effects for number of branches per plant. Female parents GP-14, GP-17, GP-18, GP-29 and GP-37 showed significant positive general combining ability effects for number of fruits per clusters. For total number of fruits per plant, female parents GP-5, GP-14, GP-32 and male parent GT-1, JT-3 reg istered as good general combiners. For average fruit weight, female parents GP-12, GP-29, GP-39 and male parents DVRT- 2 and AT- 3 showed significant positive general combining ability effects.
Female parentGP-12, GP-17, GP-29, GP-39 and male parents JT-3, DVRT-2 was considered as good general combiners for number of locules per fruit. For marketable yield per plant, female parents GP-12, GP-14, GP-32 and male parent JT-3, AT-3 were considered as good general combining ability combiners. For pericarp thickness, female parents GP-12, GP-32, GP-37, GP-39 and male parent DVRT-2, AT-3 showed significant positive general combining ability effect. For fruit shape index GP-32, GP-37 of lines and GT-1, DVRT-2, AT-3 of tester showed significant positive general combining ability effect. Female parents GP-18, GP-32 and male parent GT-1, JT-3 was considered as good general combiners for days to marketable maturity. Female parents GP-5, GP-3, GP-14, GP-32 and male parent GT-1 and JT-3 was considered as good general combiners for number of clusters per plant. Female parents GP-3, GP-5, GP-14, GP-37 and male parent GT-1, AT-3 was considered as good general combiners for total soluble solid. Whereas female parents GP-12, GP-29, GP-39 and male parents JT-3, AT-3 considered as good general combiners for titrable acidity.

As regards to specific combining ability effects (Table 3), eight crosses exhibited significant pos itive specific combining ability effects for marketable yield per plant. The highest sca effect for marketable yield per plant was exhibited by the cross GP- $5 \times$ JT-3 (poor $\times$ average) followed by GP-29 $\times$ AT-3 (poor $\times$ good), GP-18 $\times$ AT-3 (average $\times$ good). Considering the desired sca effects, the best cross combination were GP-14 $\times$ JT-3 for days to $50 \%$ flowering, GP- $14 \times$ JT-3 for days to $50 \%$ fruit set, GP-3 $\times$ DVRT- 2 for plant height, GP-12 $\times$ JT-3 for Number of branches per plant, GP-39 $\times$ JT-3 for number of fruits per cluster, GP-32 $\times$ JT-3 for Total number of fruits per plant, GP-39 $\times$ DVRT-2 for average fruit weight, GP-5 $\times$ JT-3 for number of locules per fruit, GP-18 $\times$ AT-3 for pericarp thickness, GP- $5 \times$ GT- 1 for fruit shape index, GP-12 $\times$ DVRT- 2 for days to marketable maturity and GP-37 $\times$ JT-3 for number of clusters per plant, GP-17 × DVRT-2 for total soluble solids and GP-17 × JT-3 for acidity.

\begin{tabular}{|c|c|c|c|c|c|c|c|c|c|c|c|c|c|c|c|c|}
\hline $\begin{array}{l}\text { Sr. } \\
\text { No. }\end{array}$ & Parents & $\begin{array}{c}\text { Days to } \\
\mathbf{5 0 \%} \\
\text { flowering }\end{array}$ & $\begin{array}{c}\text { Days } \\
\text { to } 50 \% \\
\text { fruit set }\end{array}$ & $\begin{array}{c}\text { Plant } \\
\text { height }\end{array}$ & $\begin{array}{c}\text { No. of } \\
\text { branches } \\
\text { per plant }\end{array}$ & $\begin{array}{l}\text { No. of } \\
\text { fruits } \\
\text { per } \\
\text { cluster }\end{array}$ & $\begin{array}{c}\text { Total } \\
\text { number } \\
\text { of fruits } \\
\text { per plant }\end{array}$ & $\begin{array}{c}\text { Average } \\
\text { fruit } \\
\text { weight }\end{array}$ & $\begin{array}{c}\text { No. of } \\
\text { locules } \\
\text { per fruit }\end{array}$ & $\begin{array}{l}\text { Market- } \\
\text { able } \\
\text { yield per } \\
\text { plant }\end{array}$ & $\begin{array}{l}\text { Pericarp } \\
\text { thickness }\end{array}$ & $\begin{array}{l}\text { Fruit } \\
\text { Shape } \\
\text { index }\end{array}$ & $\begin{array}{l}\text { Days to } \\
\text { market- } \\
\text { able } \\
\text { maturity }\end{array}$ & $\begin{array}{c}\text { No. of } \\
\text { clusters } \\
\text { per plant }\end{array}$ & $\begin{array}{c}\text { Total } \\
\text { soluble } \\
\text { solids }\end{array}$ & Acidity \\
\hline \multicolumn{17}{|c|}{ Lines } \\
\hline 1 & GP-3 & -0.967 & -1.208 & $6.207^{* *}$ & $0.355^{*}$ & $-0.900^{* *}$ & $-18.707^{*}$ & $-13.473^{* *}$ & $-0.381^{* *}$ & $-1.925^{* *}$ & $-0.460^{* *}$ & 0.019 & 0.108 & $2.553^{* *}$ & $0.840^{* *}$ & $-0.027^{* *}$ \\
\hline 2 & GP-5 & 0.533 & 0.292 & $26.09^{* *}$ & $-0.595^{* *}$ & $-0.367^{* *}$ & $31.503^{* *}$ & $-9.906^{* *}$ & 0.103 & $-0.475^{* *}$ & $-0.336^{* *}$ & $-0.063^{* *}$ & -0.142 & $8.537^{* *}$ & $0.467^{* *}$ & $-0.022^{*}$ \\
\hline 3 & GP-12 & $-1.467^{*}$ & $-1.458^{*}$ & $-8.61^{* *}$ & -0.162 & $-0.700^{* *}$ & $-24.47^{* *}$ & $12.519^{* *}$ & $0.236^{* *}$ & $1.370^{* *}$ & $0.865^{* *}$ & $-0.058^{* *}$ & -0.308 & 0.920 & 0.048 & 0.018 \\
\hline 4 & GP-14 & -0.133 & 0.125 & $15.873^{* *}$ & 0.238 & $0.400^{* *}$ & $71.737^{* *}$ & $-7.198^{* *}$ & -0.156 & $0.510^{* *}$ & $-0.447^{* *}$ & 0.001 & $1.525^{* *}$ & $12.553^{* *}$ & $0.600^{* *}$ & $0.031^{* *}$ \\
\hline 5 & GP-17 & -0.967 & -0.375 & $2.223^{* *}$ & $-1.278^{* *}$ & $1.050^{* *}$ & 9.263 & $-5.339 * *$ & $0.319^{* *}$ & -0.235 & $-0.165^{* *}$ & $-0.034^{*}$ & 0.442 & $-5.68^{* *}$ & $-0.548^{* *}$ & $-0.020^{*}$ \\
\hline 6 & GP-18 & 0.033 & -0.042 & $15.473^{* *}$ & $1.738^{* *}$ & $0.317^{*}$ & $-15.947^{*}$ & $-0.198^{*}$ & $-0.198^{*}$ & 0.236 & 0.017 & 0.02 & $-1.558^{* *}$ & $-5.83^{* *}$ & -0.036 & $-0.037^{* *}$ \\
\hline 7 & GP-29 & 0.367 & 0.625 & $-6.543^{* *}$ & $0.905^{* *}$ & $0.250^{*}$ & $-69.093^{* *}$ & $4.653^{* *}$ & $0.228^{* *}$ & $-0.390^{*}$ & $-0.289 * *$ & 0.025 & $-0.725^{* *}$ & $-16.93^{* *}$ & $-0.395^{* *}$ & $0.021^{*}$ \\
\hline 8 & GP-32 & $-2.550 * *$ & $-2.958^{* *}$ & $-1.843^{* *}$ & $-0.995^{* *}$ & 0.167 & $80.953^{* *}$ & $-2.223^{* *}$ & $-0.389 * *$ & $0.882^{* *}$ & $0.407^{* *}$ & $0.055^{* *}$ & $-2.642^{* *}$ & $14.853^{* *}$ & $-0.353^{* *}$ & 0.006 \\
\hline 9 & GP-37 & $1.450^{*}$ & $1.375^{*}$ & $-7.127^{* *}$ & $-0.312^{*}$ & $0.533^{* *}$ & 11.62 & $-4.323^{* *}$ & $-0.348^{* *}$ & -0.185 & $0.155^{* *}$ & $0.064^{* *}$ & $1.442^{* *}$ & 0.620 & $0.452^{* *}$ & -0.005 \\
\hline \multirow[t]{3}{*}{10} & GP-39 & $3.700^{* *}$ & $3.625^{* *}$ & $-41.743^{* *}$ & 0.105 & $-0.750^{* *}$ & $-76.86^{* *}$ & $25.486^{* *}$ & $0.586^{* *}$ & 0.212 & $0.252^{* *}$ & -0.028 & $1.858^{* *}$ & $-11.597^{* *}$ & $-1.075^{* *}$ & $0.034^{* *}$ \\
\hline & SE(gi) \pm & 0.645 & 0.660 & 0.697 & 0.140 & 0.130 & 7.802 & 0.097 & 0.082 & 0.162 & 0.027 & 0.017 & 0.273 & 1.011 & 0.087 & 0.010 \\
\hline & CD at $5 \%$ & 1.258 & 1.314 & 1.388 & 0.280 & 0.260 & 15.533 & 0.193 & 0.164 & 0.323 & 0.055 & 0.034 & 0.544 & 2.014 & 0.175 & 0.020 \\
\hline \multicolumn{17}{|c|}{ Testers } \\
\hline 1 & GT-1 & -0.217 & -0.242 & 0.803 & $-1.098^{* *}$ & 0.110 & $31.961^{* *}$ & $-10.733^{* *}$ & $-0.281^{* *}$ & $-0.926^{* *}$ & $-0.271^{* *}$ & $0.013^{*}$ & $-0.875^{* *}$ & $6.66^{* *}$ & $0.477^{* *}$ & $-0.032^{* *}$ \\
\hline 2 & JT-3 & -0.517 & $-1.108^{* *}$ & $-10.55^{* *}$ & $0.315^{* *}$ & -0.103 & $25.772^{* *}$ & $-1.986^{* *}$ & $0.103^{*}$ & $0.318^{* *}$ & -0.06 & -0.06 & $-1.108^{* *}$ & $6.573^{* *}$ & $-0.289 * *$ & 0.019 ** \\
\hline 3 & DVRT-2 & $-1.217^{* *}$ & $-0.875^{*}$ & $-4.477^{* *}$ & 0.062 & -0.050 & $-46.175^{* *}$ & $7.644^{* *}$ & $0.136^{* *}$ & $-0.426^{* *}$ & $0.157^{* *}$ & $0.013^{*}$ & $0.392^{*}$ & $-8.84^{* *}$ & $-0.345^{* *}$ & -0.007 \\
\hline \multirow[t]{3}{*}{4} & AT-3 & $1.95^{* *}$ & $2.225^{* *}$ & $14.223^{* *}$ & $0.722^{* *}$ & 0.043 & $-11.559 *$ & $5.074^{* *}$ & 0.043 & $1.034^{* *}$ & $0.174^{* *}$ & $0.034^{* *}$ & $1.592^{* *}$ & $-4.393^{*}$ & $0.157^{* *}$ & $0.020^{* *}$ \\
\hline & $\mathrm{SE}($ gi) \pm & 0.408 & 0.417 & 0.441 & 0.089 & 0.082 & 4.934 & 0.061 & 0.052 & 0.102 & 0.017 & 0.010 & 0.173 & 0.639 & 0.055 & 0.006 \\
\hline & $\mathrm{CD}$ at $5 \%$ & 0.813 & 0.831 & 0.878 & 0.177 & 0.164 & 9.824 & 0.122 & 0.104 & 0.204 & 0.035 & 0.021 & 0.344 & 1.273 & 0.110 & 0.01 \\
\hline
\end{tabular}

Table 2: General combining ability effects for different characters in tomato*, ${ }^{* *}$ Significant at $5 \%$ and $1 \%$ against error, respectively 


\begin{tabular}{|c|c|c|c|c|c|c|c|c|c|}
\hline $\begin{array}{l}\text { Sr. } \\
\text { No. }\end{array}$ & Hybrids & $\begin{array}{c}\text { Days to } \\
50 \% \\
\text { flowering }\end{array}$ & $\begin{array}{c}\text { Days to } \\
50 \% \\
\text { fruit set }\end{array}$ & $\begin{array}{l}\text { Plant } \\
\text { height }\end{array}$ & $\begin{array}{c}\text { No. of } \\
\text { branches } \\
\text { per plant }\end{array}$ & $\begin{array}{l}\text { No. of } \\
\text { fruits per } \\
\text { cluster }\end{array}$ & $\begin{array}{c}\text { Total number } \\
\text { of fruits per } \\
\text { plant }\end{array}$ & $\begin{array}{l}\text { Aver- } \\
\text { age fruit } \\
\text { weight }\end{array}$ & $\begin{array}{c}\text { No. of } \\
\text { locules per } \\
\text { fruit }\end{array}$ \\
\hline 1 & GP-3 $\times$ GT-1 & -1.7 & -2.092 & $-21.52^{* *}$ & 0.532 & -0.293 & $-32.701^{*}$ & $13.033^{* *}$ & $1.131^{* *}$ \\
\hline 2 & GP-3 $\times$ JT-3 & 1.267 & 1.442 & $6.3^{* *}$ & $-1.548^{* *}$ & $1.453^{* *}$ & $66.782^{* *}$ & $1.219^{* *}$ & $-0.519^{* *}$ \\
\hline 3 & GP-3 $\times$ DVRT- 2 & 1.967 & 1.542 & $46.027^{* *}$ & 0.305 & $-0.600 *$ & 16.768 & $-2.478^{* *}$ & -0.219 \\
\hline 4 & GP-3 $\times$ AT- 3 & -1.533 & -0.892 & $-30.807^{* *}$ & $0.712^{*}$ & $-0.560 *$ & $-50.848^{* *}$ & $-11.774^{* *}$ & $-0.393^{*}$ \\
\hline 5 & GP-5 $\times$ GT-1 & -0.533 & -0.925 & $-16.47^{* *}$ & $-0.585^{*}$ & $0.640^{*}$ & $81.502^{* *}$ & -0.001 & $-1.153^{* *}$ \\
\hline 6 & GP-5 × JT-3 & 2.1 & 1.275 & $-6.517^{* *}$ & $1.135^{* *}$ & 0.053 & $44.638^{* *}$ & $19.453^{* *}$ & $1.664^{* *}$ \\
\hline 7 & GP-5 $\times$ DVRT- 2 & 0.133 & 0.042 & $14.61^{* *}$ & $2.522^{* *}$ & $0.800^{*}$ & 9.011 & $-12.378^{* *}$ & $-0.569 * *$ \\
\hline 8 & GP-5 × AT-3 & -1.7 & -0.392 & $8.377^{* *}$ & $-3.072^{* *}$ & $-1.493^{* *}$ & $-135.15^{* *}$ & $-7.074^{* *}$ & 0.057 \\
\hline 9 & GP-12 × GT- 1 & 1.8 & 2.158 & $22.297^{* *}$ & $0.782^{* *}$ & -0.493 & -8.138 & 0.108 & 0.248 \\
\hline 10 & GP-12 $\times$ JT-3 & -0.567 & -1.642 & $7.983^{* *}$ & $2.768^{* *}$ & $0.920^{* *}$ & -14.815 & $2.494^{* *}$ & 0.264 \\
\hline 11 & GP-12 × DVRT-2 & -1.867 & -1.208 & $-12.69 * *$ & $-3.178^{* *}$ & -0.267 & $52.638^{* *}$ & $-15.903^{* *}$ & -0.303 \\
\hline 12 & GP-12 × AT-3 & 0.633 & 0.692 & $-17.59 * *$ & -0.372 & -0.160 & -29.685 & $13.301^{* *}$ & -0.209 \\
\hline 13 & GP-14 × GT-1 & 1.467 & 0.908 & $8.28^{* *}$ & 0.382 & $-0.593^{*}$ & $39.402^{*}$ & $1.458^{* *}$ & -0.061 \\
\hline 14 & GP-14 × JT-3 & $-3.233^{*}$ & $-3.558^{* *}$ & 0.7 & $-1.832^{* *}$ & $-0.780^{* *}$ & $-69.755^{* *}$ & $5.011^{* *}$ & $0.456^{* *}$ \\
\hline 15 & GP-14 $\times$ DVRT- 2 & 0.467 & 1.208 & $2.827^{*}$ & -0.112 & 0.300 & -8.329 & $-4.519 * *$ & 0.289 \\
\hline 16 & GP-14 × AT-3 & 1.3 & 1.442 & $-11.807^{* *}$ & $1.562^{* *}$ & $1.073^{* *}$ & $38.682^{*}$ & $-1.949 * *$ & $-0.684^{* *}$ \\
\hline 17 & GP-17 × GT-1 & -2.033 & -0.258 & $-23.403^{* *}$ & $-1.835^{* *}$ & $0.690^{* *}$ & 28.742 & $8.199^{* *}$ & 0.164 \\
\hline 18 & GP-17 × JT-3 & -0.4 & -2.058 & $14.95^{* *}$ & -0.248 & 0.437 & $-34.548^{*}$ & $-3.281^{* *}$ & $-0.352^{*}$ \\
\hline 19 & GP-17 $\times$ DVRT-2 & 1.633 & 2.042 & $4.077^{* *}$ & -0.062 & $-1.017^{* *}$ & -4.829 & $-4.611^{* *}$ & $-0.453^{* *}$ \\
\hline 20 & GP-17 × AT-3 & 0.8 & 0.275 & $4.377^{* *}$ & $2.145^{* *}$ & -0.110 & 10.635 & -0.307 & $0.641^{* *}$ \\
\hline 21 & GP-18 $\times$ GT-1 & 1.3 & 0.408 & $20.347^{* *}$ & $1.015^{* *}$ & 0.357 & $-30.594^{*}$ & $-6.176^{* *}$ & -0.119 \\
\hline 22 & GP-18 × JT-3 & -0.067 & 1.275 & $-17.833^{* *}$ & 0.335 & $-2.23^{* *}$ & $-92.074^{* *}$ & $6.878^{* *}$ & -0.236 \\
\hline 23 & GP-18 $\times$ DVRT- 2 & -0.367 & 0.042 & $-35.44^{* *}$ & $-1.012^{* *}$ & $0.650^{*}$ & $32.755^{*}$ & $-8.719^{* *}$ & -0.136 \\
\hline 24 & GP-18 $\times$ AT- 3 & -0.867 & -1.725 & $32.927^{* *}$ & -0.338 & $1.223^{* *}$ & $89.913^{* *}$ & $8.018^{* *}$ & $0.491^{* *}$ \\
\hline 25 & GP-29 $\times$ GT-1 & 1.633 & 1.742 & $-4.637^{* *}$ & $2.115^{* *}$ & $-1.310^{* *}$ & $-57.381 * *$ & $-9.959^{* *}$ & $-0.578^{* *}$ \\
\hline 26 & GP-29 × JT-3 & 0.267 & 0.942 & $-15.083^{* *}$ & $1.102^{* *}$ & $-1.163^{* *}$ & $-60.645^{* *}$ & $-6.539 * *$ & -0.128 \\
\hline 27 & GP-29 $\times$ DVRT-2 & -0.7 & -1.292 & $3.377^{*}$ & $-0.912^{* *}$ & $1.250^{* *}$ & 14.528 & $4.798^{* *}$ & 0.306 \\
\hline 28 & GP-29 $\times$ AT- 3 & -1.2 & -1.392 & $16.343^{* *}$ & $-2.305^{* *}$ & $1.223^{* *}$ & $103.499^{* *}$ & $11.701^{* *}$ & $0.399 *$ \\
\hline 29 & GP-32 × GT- 1 & -1.45 & -1.008 & $27.863^{* *}$ & $-1.652^{* *}$ & -0.493 & $37.945^{*}$ & $3.683^{* *}$ & $0.373^{*}$ \\
\hline 30 & GP-32 × JT-3 & 0.85 & 0.858 & $43.15^{* *}$ & -0.265 & $1.12^{* *}$ & $141.362^{* *}$ & $-10.898^{* *}$ & $-1.044^{* *}$ \\
\hline 31 & GP-32 $\times$ DVRT- 2 & -0.783 & -1.375 & $-14.59 * *$ & $2.388^{* *}$ & 0.333 & $-74.919^{* *}$ & $6.373^{* *}$ & $0.523^{* *}$ \\
\hline 32 & GP-32 $\times$ AT- 3 & 1.383 & 1.525 & $-56.423^{* *}$ & -0.472 & $-0.96^{* *}$ & $-104.39 * *$ & $0.842^{* *}$ & 0.149 \\
\hline 33 & GP-37 × GT-1 & 0.883 & 1.325 & $-9.453^{* *}$ & $-1.402^{* *}$ & $0.607^{*}$ & $-40.695^{* *}$ & $7.016^{* *}$ & 0.231 \\
\hline 34 & GP-37 × JT-3 & -0.817 & -0.475 & $5.700^{* *}$ & $0.918^{* *}$ & $-1.447^{* *}$ & 29.815 & $-2.398^{* *}$ & -0.086 \\
\hline 35 & GP-37 × DVRT- 2 & 0.217 & -0.042 & $-30.773^{* *}$ & -0.295 & -0.100 & $-51.105^{* *}$ & 0.339 & 0.081 \\
\hline 36 & GP-37 $\times$ AT-3 & -0.283 & -0.808 & $34.527^{* *}$ & $0.778^{* *}$ & $0.940^{* *}$ & $61.985^{* *}$ & $-4.958^{* *}$ & -0.226 \\
\hline 37 & GP-39 × GT-1 & -1.367 & -2.258 & $-3.303^{*}$ & $0.648^{*}$ & $0.890^{* *}$ & -18.081 & $-17.359 * *$ & -0.236 \\
\hline 38 & GP-39 × JT-3 & 0.600 & 1.942 & $-39.35^{* *}$ & $-2.365^{* *}$ & $1.637^{* *}$ & -10.758 & $-11.939 * *$ & -0.019 \\
\hline 39 & GP-39 × DVRT-2 & -0.700 & -0.958 & $22.577^{* *}$ & 0.355 & $-1.35^{* *}$ & 13.481 & $37.098^{* *}$ & $0.481^{* *}$ \\
\hline \multirow[t]{3}{*}{40} & GP-39 × AT-3 & 1.467 & 1.275 & $20.077^{* *}$ & $1.362^{* *}$ & $-1.177^{* *}$ & 15.359 & $-7.799^{* *}$ & -0.226 \\
\hline & SE \pm & 1.291 & 1.320 & 1.395 & 0.281 & 0.261 & 15.605 & 0.194 & 0.165 \\
\hline & CD at $5 \%$ & 2.571 & 2.629 & 2.777 & 0.561 & 0.521 & 31.067 & 0.386 & 0.329 \\
\hline
\end{tabular}




\begin{tabular}{|c|c|c|c|c|c|c|c|c|}
\hline $\begin{array}{c}\text { Sr. } \\
\text { No. }\end{array}$ & Hybrids & $\begin{array}{c}\text { Marketable } \\
\text { yield per plant }\end{array}$ & $\begin{array}{c}\text { Pericarp } \\
\text { thickness }\end{array}$ & $\begin{array}{c}\text { Fruit shape } \\
\text { index }\end{array}$ & $\begin{array}{c}\text { Days to } \\
\text { marketable } \\
\text { maturity }\end{array}$ & $\begin{array}{c}\text { No. of } \\
\text { clusters per } \\
\text { plant }\end{array}$ & $\begin{array}{c}\text { Total } \\
\text { Soluble } \\
\text { Solids }\end{array}$ & Acidity \\
\hline 1 & GP-3 $\times$ GT-1 & $1.205^{* *}$ & $0.784^{* *}$ & -0.051 & -0.875 & -3.160 & -0.267 & 0.038 \\
\hline 2 & GP-3 3 JT-3 & $0.688^{*}$ & $0.313^{* *}$ & 0.016 & $-1.308^{*}$ & 2.460 & 0.306 & $-0.040^{*}$ \\
\hline 3 & GP-3 $\times$ DVRT- 2 & 0.592 & $-0.113^{*}$ & $0.073^{*}$ & $2.192^{* *}$ & $9.473^{* *}$ & 0.268 & 0.001 \\
\hline 4 & GP-3 × AT-3 & $-2.485^{* *}$ & $-0.984^{* *}$ & -0.038 & -0.008 & $-8.773^{* *}$ & -0.307 & 0.002 \\
\hline 5 & GP-5 × GT-1 & -0.612 & $-0.506^{* *}$ & $0.111^{* *}$ & $-1.958^{* *}$ & $11.79^{* *}$ & $0.460^{* *}$ & 0.019 \\
\hline 6 & GP-5 × JT-3 & $5.438^{* *}$ & $0.717^{* *}$ & $-0.259^{* *}$ & 0.275 & $14.41^{* *}$ & $-2.254^{* *}$ & $-0.039 *$ \\
\hline 7 & GP-5 × DVRT- 2 & $-1.085^{* *}$ & $-0.117^{*}$ & $0.085^{*}$ & 0.442 & $-4.043^{*}$ & $0.388^{*}$ & -0.003 \\
\hline 8 & GP-5 × AT-3 & $-3.741^{* *}$ & -0.094 & 0.063 & $1.242^{*}$ & $-22.157^{* *}$ & $1.407^{* *}$ & 0.023 \\
\hline 9 & GP-12 × GT-1 & 0.371 & -0.040 & -0.037 & -0.458 & $4.007^{*}$ & $0.658^{* *}$ & 0.012 \\
\hline 10 & GP-12 $\times$ JT-3 & 0.457 & $0.726^{* *}$ & 0.033 & $3.775^{* *}$ & $-11.373^{* *}$ & $0.398 *$ & $-0.079^{* *}$ \\
\hline 11 & GP-12 × DVRT- 2 & -0.279 & $-1.014^{* *}$ & 0.040 & $-4.058^{* *}$ & $13.573^{* *}$ & $-0.347^{*}$ & 0.004 \\
\hline 12 & GP-12 × AT-3 & -0.549 & $0.328^{* *}$ & -0.035 & 0.742 & $-6.207^{* *}$ & $-0.709^{* *}$ & $0.063^{* *}$ \\
\hline 13 & GP-14 × GT-1 & -0.322 & $-0.398^{* *}$ & 0.064 & -0.958 & $13.84^{* *}$ & $-0.56^{* *}$ & $-0.044^{*}$ \\
\hline 14 & GP-14 × JT-3 & -0.373 & $0.207^{* *}$ & -0.052 & 0.608 & $-7.873^{* *}$ & $0.799^{* *}$ & $0.065^{* *}$ \\
\hline 15 & GP-14 × DVRT- 2 & 0.148 & $0.291^{* *}$ & -0.025 & 0.775 & $-4.993^{*}$ & $-0.626^{* *}$ & $0.085^{* *}$ \\
\hline 16 & GP-14 × AT-3 & 0.548 & -0.100 & 0.013 & -0.425 & -0.973 & $0.386^{*}$ & $-0.106^{* *}$ \\
\hline 17 & GP-17 × GT-1 & $1.696^{* *}$ & $0.206^{* *}$ & 0.018 & $2.458^{* *}$ & -0.860 & $-1.279^{* *}$ & -0.026 \\
\hline 18 & GP-17 × JT-3 & $-1.315^{* *}$ & $0.125^{*}$ & 0.025 & $-2.642^{* *}$ & $-9.707^{* *}$ & $-0.599^{* *}$ & $0.110^{* *}$ \\
\hline 19 & GP-17 × DVRT- 2 & -0.231 & 0.029 & -0.024 & 0.192 & $5.507^{* *}$ & $1.930^{* *}$ & -0.038 \\
\hline 20 & GP-17 × AT-3 & -0.150 & $-0.359 * *$ & -0.019 & -0.008 & $5.060^{*}$ & -0.052 & $-0.045^{*}$ \\
\hline 21 & GP-18 × GT- 1 & $-1.772^{* *}$ & $-0.989 * *$ & -0.025 & $2.458^{* *}$ & $-9.577^{* *}$ & 0.243 & -0.026 \\
\hline 22 & GP-18 × JT-3 & $-1.849^{* *}$ & $0.123^{*}$ & 0.058 & -0.975 & -3.957 & -0.258 & $-0.047^{*}$ \\
\hline 23 & GP-18 × DVRT- 2 & -0.255 & $-0.223^{* *}$ & -0.018 & -0.475 & 2.923 & -0.149 & $-0.044^{*}$ \\
\hline 24 & GP-18 $\times$ AT-3 & $3.876^{* *}$ & $1.089^{* *}$ & -0.016 & -1.008 & $10.610^{* *}$ & 0.163 & $0.118^{* *}$ \\
\hline 25 & GP-29 × GT- 1 & $-1.852^{* *}$ & $0.157^{* *}$ & -0.007 & 0.292 & $-6.543^{* *}$ & $1.422^{* *}$ & -0.017 \\
\hline 26 & GP-29 × JT-3 & $-2.443^{* *}$ & $-0.568^{* *}$ & 0.053 & $-1.142 *$ & $-8.857^{* *}$ & $-1.166^{* *}$ & -0.031 \\
\hline 27 & GP-29 × DVRT- 2 & -0.249 & $0.196^{* *}$ & -0.043 & -0.642 & -0.310 & $-0.371^{*}$ & -0.005 \\
\hline 28 & GP-29 $\times$ AT-3 & $4.545^{* *}$ & $0.215^{* *}$ & -0.004 & $1.492^{* *}$ & $15.710^{* *}$ & 0.115 & $0.054^{* *}$ \\
\hline 29 & GP-32 × GT- 1 & $1.669^{* *}$ & $0.814^{* *}$ & -0.037 & 0.542 & $15.740^{* *}$ & 0.193 & 0.024 \\
\hline 30 & GP-32 × JT-3 & -0.289 & $-1.247^{* *}$ & 0.036 & $2.775^{* *}$ & $14.093^{* *}$ & $1.613^{* *}$ & -0.030 \\
\hline 31 & GP-32 $\times$ DVRT- 2 & 0.469 & $0.244^{* *}$ & $0.080^{*}$ & -0.725 & $-17.427^{* *}$ & $-0.892^{* *}$ & 0.020 \\
\hline 32 & GP-32 × AT-3 & $-1.848^{* *}$ & $0.189 * *$ & $-0.078^{*}$ & $-2.592^{* *}$ & $-12.407^{* *}$ & $-0.914^{* *}$ & -0.015 \\
\hline 33 & GP-37 × GT-1 & 0.349 & $0.3^{* *}$ & -0.019 & 0.458 & $-14.893^{* *}$ & $-0.458^{* *}$ & 0.005 \\
\hline 34 & GP-37 × JT-3 & 0.158 & $0.276^{* *}$ & 0.001 & $-3.642^{* *}$ & $23.06^{* *}$ & $0.761^{* *}$ & 0.035 \\
\hline 35 & GP-37 $\times$ DVRT- 2 & $-0.878^{* *}$ & $-0.297^{* *}$ & -0.002 & $1.858^{* *}$ & $-12.327^{* *}$ & $-0.497^{* *}$ & 0.024 \\
\hline 36 & GP-37 × AT-3 & 0.372 & $-0.278^{* *}$ & 0.020 & $1.325^{*}$ & $4.160^{*}$ & 0.195 & $-0.064^{* *}$ \\
\hline 37 & GP-39 × GT-1 & $-0.731^{*}$ & $-0.328^{* *}$ & -0.017 & $-1.958^{* *}$ & $-10.343^{* *}$ & $-0.412^{*}$ & 0.016 \\
\hline 38 & GP-39 × JT-3 & -0.469 & $-0.672^{* *}$ & $0.089 * *$ & $2.275^{* *}$ & $-12.257^{* *}$ & $0.401^{*}$ & $0.055^{* *}$ \\
\hline 39 & GP-39 $\times$ DVRT- 2 & $1.769^{* *}$ & $1.005^{* *}$ & $-0.167^{* *}$ & 0.442 & $7.623^{* *}$ & 0.296 & $-0.042 *$ \\
\hline \multirow[t]{3}{*}{40} & GP-39 × AT-3 & -0.568 & -0.006 & $0.095^{* *}$ & -0.758 & $14.977^{* *}$ & -0.285 & -0.030 \\
\hline & $\mathrm{SE} \pm$ & 0.324 & 0.055 & 0.034 & 0.547 & 2.023 & 0.175 & 0.020 \\
\hline & CD at $5 \%$ & 0.646 & 0.111 & 0.068 & 1.089 & 4.028 & 0.349 & 0.040 \\
\hline
\end{tabular}

Table 3: Specific combining ability effects of various characters in tomato*, ${ }^{* *}$ Significant at $5 \%$ and $1 \%$ against error, respectively. 
The gca effects of the parents and sca effects of their crosses in the present study indicated that the crosses between two high general combiners were not always the best their sca effects. The best specific cross combinations for different characters in present study were the combinations of good $\times$ good, good $\times$ average, average $x$ average, average $\times$ poor, poor $\times$ poor and good $\times$ poor general combiners. The marked desirable specific combining ability effects in crosses between poor $\times$ poor combiners includes, GP-3 $\times$ GT- 1 for number of locules per fruit, GP-5 $\times$ JT-3 for average fruit weight; poor $\times$ average combiner e.g., GP-39 $\times$ JT-3 for number of fruits per cluster; average $\times$ average combiner e.g., GP-14 $\times$ JT-3 Days to $50 \%$ flowering; average $\times$ good e.g., GP- $14 \times$ JT- 3 for days to $50 \%$ fruit set, GP-12 $\times$ JT-3 for number of branches per plant; good $\times$ good e.g., GP-39 $\times$ DVRT- 2 for average fruit weight and GP-32 $\times$ JT-3 for total number of fruits per plant, GP-12 $\times$ AT- 3 for average fruit weight; good $\times$ poor e.g., GP-3 $\times$ DVRT- 2 for plant height.

In fact, in majority of cases, the best specific combinations for different characters were either poor $\times$ poor, good $\times$ poor, average $\times$ poor, average $\times$ average and vise versa general combiners. This suggested that information on gca effects should be supplemented by sca effects and hybrid performance of cross combinations to predict the transgressive type possibly made available in segregating generations. Selection is rapid if gca effects of parents and sca effects of crosses are in same direction. If crosses showing high sca effects involve at least one parent possessing good gca effect and high mean value, they could be exploited for practical breeding. However, high sca effects would not necessarily mean a high performance by the hybrid and the estimation of sca effects seemed to be superfluous, as no additional information was obtained by doing so. Therefore, it is suggested that the selection of parents for further breeding programme should be based on gca effects and due consideration should be given to mean value of the cross combinations while selecting crosses for specific combining ability effects.

\section{Bibliography}

1. Tiwari RN and Choudhury B. "Tomato. In: Solanaceous Crops". (Eds.): B. Som \& K. N. Prokash. Calcutta (1986): 224-280.

2. Sharma P., et al. "Combining ability for certain quality traits in bacterial wilt resistant genotypes in tomato". Environment and Ecology 24.1 (2006): 102-105.

3. Ahmed S., et al. "Combining ability estimates of tomato (Solanum lycopersicum L.) in late summer". SAARC Journal of Agriculture 7.1 (2009): 43-55.
4. Singh B., et al. "Combining ability for yield and its contributing characters in tomato". Indian Journal of Horticulture 67 (2010): 50-55.

5. Gaikwad SP., et al. "Combining ability analysis in tomato by use of line x tester technique". Journal of Maharastra Agricultural University 27.3 (2002): 308-310.

6. Mali and Patel AI. "Heterosis study in tomato (Lycopersicon esculentum Mill.)". Trends in Biosciences 7.4 (2014): 250-253.

7. Angadi A., et al. "Combining ability studies for productivity related traits in tomato (Lycopersicon esculentum Mill.)". asian journal of horticulture 7.1 (2012): 118-122.

8. Yadav SK., et al. "Genetic study of heterosis for yield and quality components in tomato (Solanum Lycopersicum)". African Journal of Agricultural Research 8.44 (2013): 5585-5591.

9. Agarwal A., et al. "Heterosis, combining ability and gene action for yield and quality traits in tomato (Solanum lycopersicum L.)". Helix 2.3 (2014): 511-515.

10. EI-Gabry MAH., et al. "Combining ability and heritability of some tomato (Solanum lycopersicum L.)". Scientia Horticulturae 167 (2014): 153-157.

11. Singh AK and Asati BS. "Combining ability and heterosis studies in tomato under bacterial wilt condition". Bangladesh Journal of Agricultural Research 36.2 (2011): 313-318.

12. Kumari S and Sharma KM. "Line $\mathrm{x}$ tester analysis to study combining ability effects in tomato (Solanum lycopersicum L.)". Vegetable Science 39.1 (2012): 65-69.

13. Souza LM., et al. "Diallel cross among fresh market tomato inbreebing lines”. Horticulture Brasileira 30.2 (2012): 246-251.

14. Shankar A., et al. "Combining ability and gene action studies for yield and yield contributing traits in tomato (Solanum Lycopersicum L.)". Helix 6.2 (2013): 431-435.

15. Tanvi R., et al. "Combining ability and geneaction studies in some genetic stocks of tomato (Solanum lycopersicum L.)". International Journal of Current Microbiology and Applied Sciences 6.10 (2017): 138-144.

Volume 3 Issue 7 July 2019

(C) All rights are reserved by TA Vekariya, et al. 